\title{
CENTRALIDADE DE GRAFOS APLICADA À PROJETOS DE ENERGIA RENOVÁVEL
}

\section{GRAPHICS CENTRALITY APPLIED TO RENEWABLE ENERGY PROJECTS}

\author{
LETÍCIA FERNANDES BELLA GOMES, M.SC. | UFF \\ KARINE FÁTIMA DE FREITAS | UFF \\ RICARDO LUIZ FERNANDES BELLA, Dr. | UFF \\ RENATA RAPOSO DEL-VECCHIO, Dra. | UFF \\ GILSON BRITO ALVES LIMA, Dr. | UFF
}

\begin{abstract}
RESUMO
Este estudo visa aplicar a teoria de grafos através da medida de centralidade de autovetor em atividades macro (EAP) de projetos de construção de usinas geradoras de energia elétrica de fonte renovável como eólica, solar, hídrica e biomassa. Os subprojetos que compõem o projeto principal, também chamados na metodologia do PMI como atividades de nível 1, são baseados nos cinco escopos principais para a construção de uma usina elétrica: (1) Projeto Civil, (2) Projeto Elétrico de Subestação, (3) Projeto Elétrico de Linha de Rede de Média Tensão, (4) Projeto Elétrico de Linha de Alta Tensão/ Transmissão, (5) Projeto de Aquisição de Maquinário Especíico. A relação de impacto em cronograma final configurada por atraso em um dos subprojetos analisados par a par é analisada como a ligação entre os vértices do grafo traçado para estudo. Os resultados finais obtidos através da aplicação do método são analisados e evidenciam a aplicabilidade da teoria de grafos em diversos segmentos de mercado desde que se possa estabelecer conceitos de vértices e relação entre os mesmos.
\end{abstract}

PALAVRAS CHAVE: Teoria de Grafos, Centralidade, Projeto

This study aims to apply the theory of graphs through the measure of eigenvector centrality in macro projects activities (WBS) of construction of electrical plants of renewable sources such as wind, solar, hydro and biomass. The subprojects that make up the main project, also called by PMI methodology as level 1 activities, are based on the five main scopes for the construction project: (1) Civil Project, (2) Substation Electrical Project, (3) Medium Voltage Electrical Project, (4) High Voltage Electrical Project, (5) Project of Acquisition of Specific Machinery. The final timing impact relation set by delay in one of the subprojects considering other subproject delays is analyzed as the link between the vertices of the plotted graph for study. The final result obtained through the application of the method are analyzed and evidence the applicability of the theory of graphs in several market segments since it is possible to establish concepts of vertices and connection relation between them.
\end{abstract}

KEYWORDS: Graph theory, Centrality, Project 


\section{INTRODUÇÃO}

Um projeto de construção de usina geradora de energia elétrica de fonte renovável, assim como projetos em geral, possui uma Estrutura Analítica de Projetos - EAP de atividades a serem realizadas a fim de que o escopo do projeto seja cumprido conforme planejamento de prazo, custo e requisitos especificados de conteúdo e qualidade.

Neste estudo trataremos da EAP macro composta pelas atividades principais de uma obra de tal porte, que são: (1) Projeto Civil, (2) Projeto Elétrico de Subestação, (3) Projeto Elétrico de Linha de Rede de Média Tensão, (4) Projeto Elétrico de Linha de Alta Tensão/ Transmissão, (5) Projeto de Aquisição de Maquinário Específico.

As etapas macro do projeto de construção de usina geradora de energia elétrica de fonte renovável também podem ser consideradas como subprojetos do projeto principal. Por se tratarem de etapas de um projeto são executadas em paralelo, porém possuem inter-relação de prazo. Ou seja, um atraso em um subprojeto pode acarretar em atrasos em outros subprojetos dependendo dos requisitos de entrada e saída de cada um deles.

Abordaremos a relação de interdependência dos projetos através de um grafo cujos vértices são os subprojetos e as arestas se dão no caso de haver relação de impacto em cronograma final dado um atraso em um dos subprojetos analisados pares a par.

O objetivo final deste estudo é identificar, através do cálculo de centralidade de autovetor do grafo do problema detalhado acima, qual o subprojeto responsável pela maior propagação de atrasos no cronograma final do projeto principal dado um atraso específico no mesmo. Assim, proporcionando a um gerente de projeto de construção de usina geradora de energia elétrica de fonte renovável dados para maior controle e prevenção de atrasos em tal etapa, mitigando os atrasos totais possíveis em seu projeto.

\section{REVISÃO BIBLIOGRÁFICA}

\subsection{Grafos}

Grafos são redes compostas por um conjunto de vértices e arestas, sendo as arestas a representação gráfica da relação entre dois vértices (BONDY; MURTY, 1976).

Essa relação entre dois vértices dada pelas arestas é chamada adjacência ao se expressar a relação gráfica através de Matrizes (BORBA, 2013).
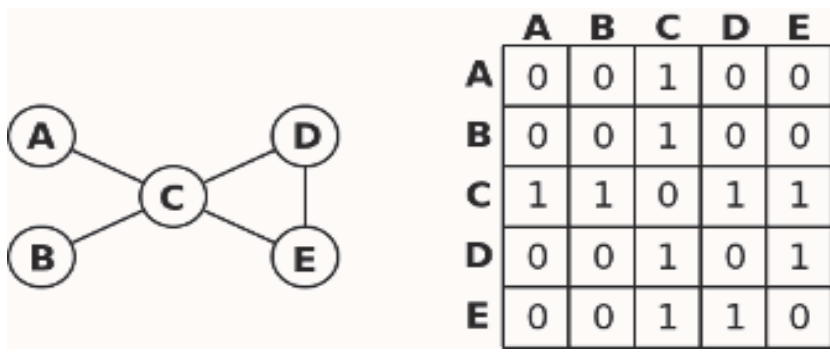

Figura 01 - Exemplo de grafo e sua matriz adjacência

Fonte: http://wikiciencias.casadasciencias.org/wiki/index.php/Grafo

Trataremos, neste estudo, de grafos conexos, simples e não-direcionados. Um grafo é dito conexo se em um grafo $G(V, E)$ existe um caminho entre qualquer par de vértices, onde $n=|V|$ e $v_{i} \in V$ (BEAUCHAMP, 1965).

Centralidade é uma medida realizada nos grafos tendo em vista sua característica de posição que visa medir o quão central cada vértice é em relação à rede, ou seja, mede a importância de um determinado vértice em relação à sua posição na rede (BORGATTI, 2005; BONACICH, 1987).

Freeman (1979) apresenta três medidas clássicas para cálculo de centralidade: centralidade de grau, centralidade de intermediação e centralidade de autovetor (DE FREITAS, 2010).

\subsubsection{Centralidade de Grau}

A centralidade é entendida como o número de relações ou conexões diretas de um vértice com os demais (DELVECCHIO et al., 2009). Assim, a centralidade de grau de um vértice mede a quantidade de ligações que um dado vértice possui com outros vértices da rede, ou seja, o número de arestas que o ligam aos demais, tratando de grafos simples, sem laços e não direcionados. Portanto, o grau de um vértice pode ser expresso também na matriz adjacência do grafo, sendo esta a expressão da relação das ligações entre os vértices de um grafo. Sendo assim, o grau (di) de um vértice i é definido por (NETTO, 2003):

$$
d i=\sum_{(f=1)}^{n} a_{i f}
$$

Onde $a_{\text {if }}$ são os elementos da matriz de adjacência.

\subsubsection{Centralidade de Intermediação}

A centralidade de intermediação de um vértice mede a influência de um dado vértice nos demais da rede, influência esta medida pelo fato de que este vértice faz parte do caminho entre dois outros do grafo. Seja $\sigma\left(v_{j} v_{k}\right)\left(v_{j}\right) o$ número de menores caminhos que ligam os vértices $v_{j} e$ $v_{k}$ passando por $v_{i^{\prime}}$ e $\sigma\left(v_{j} v_{k}\right)$ o total de caminhos mínimos entre $v_{j}$ e $v_{k^{\prime}} \operatorname{com} v_{j} v_{k} \in V$ e $v_{j} \neq v_{k} \neq v_{i^{\prime}}$, ela pode ser calculada pela equação (2) a seguir (DEL-VECCHIO et al., 2009): 


$$
c_{B}\left(v_{i}\right)=\sum_{v_{j} \neq v_{k} \neq v_{i}, v_{j} \neq v_{i}}^{v_{j} v_{k}} \frac{\sigma_{v_{j} v_{k}}\left(v_{i}\right)}{\sigma_{v_{j} v_{k}}}
$$

Portanto, a frequência com que um vértice se situa entre dois outros vértices do grafo, tomando como referência o menor caminho entre eles, é a centralidade de intermediação de tal vértice do grafo.

\subsubsection{Centralidade de Autovetor}

A centralidade de autovetor mede a importância de um vértice dadas as importâncias dos seus vizinhos (BONACICH, 1987; DE FREITAS, 2010). Isto significa que mesmo um vértice cujo grau não é elevado em relação aos outros pode ter sua centralidade elevada caso seus vizinhos possuam grau elevado.

Através da representação de um grafo por matriz, pode-se se dizer também que esta medida corresponde às coordenadas do autovetor associado ao maior autovalor da matriz de adjacência do grafo (DEL-VECCHIO et al., 2009).
Portanto, a centralidade de autovetor mede a conexão de um vértice a outros com elevada centralidade de grau. A centralidade (Ceig) do vértice vi é dada por:

$$
C_{e i g_{i}}=x_{i}
$$

Onde xi é a i-ésima coordenada do autovetor positivo unitário $x$ associado ao maior autovalor da matriz de adjacência, isto é, $x$ satisfaz a equação matricial $A x=\lambda x$, onde $\lambda$ é o maior autovalor de $A$.

\subsection{Projetos}

Gerenciamento de projetos é definido pelo instituto de referência no tema, o PMI, como a aplicação de conhecimentos e ferramentas para se planejar, executar e controlar projetos de forma eficiente e eficaz; sendo projetos entendido como um evento único com prazo definido de início e fim (PMBOK, 2004). (SLACK et al, 2009) define projeto como um conjunto de atividades, que tem um ponto inicial e um estado final definidos, persegue uma meta definida e usa um conjunto de recursos.

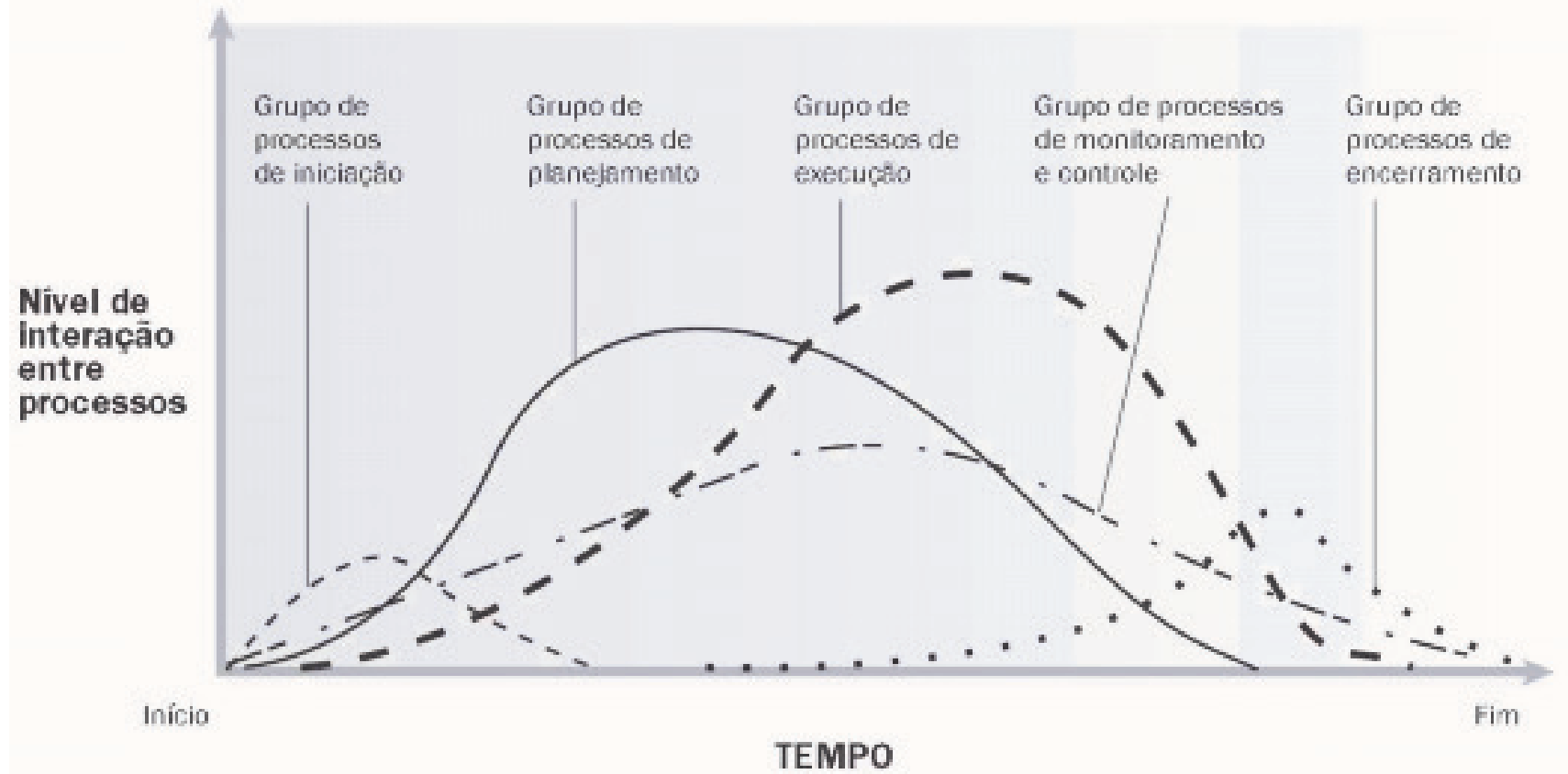

Figura 02 - Processos de um projeto

Fonte: PMI (2013)

As etapas e atividades que compõem um projeto são detalhadas e acompanhadas através da EAP Estrutura Analítica do Projeto. Dado que as atividades de um projeto são realizadas de forma sequencial e até mesmo realizadas de forma paralela em alguns casos, pode-se afirmar que as mesmas possuem uma relação de sucessão (atividades predecessoras e atividades sucessoras). Essas etapas também podem ser agrupadas de acordo com sua semelhança e interdependência em níveis ou subprojetos.

Em um projeto de construção de usina geradora de energia elétrica de fonte renovável, por exemplo, pode-se agrupar as atividades e etapas da construção em função do escopo macro principal das atividades, sendo eles: (1) Projeto Civil; (2) Projeto Elétrico de Subestação; (3) Projeto Elétrico de Linha de Rede de Média Tensão; (4) Projeto 
Elétrico de Linha de Alta Tensão/ Transmissão; (5) Projeto de Aquisição de Maquinário Específico. (BRACIANI, 2011). A relação entre as atividades e subprojetos de um grafo podem ser vistas de modo equivalente a relação de ligação/ arestas entre os vértices de um grafo (GRASSI; STEFANI; TORRIERO, 2010).

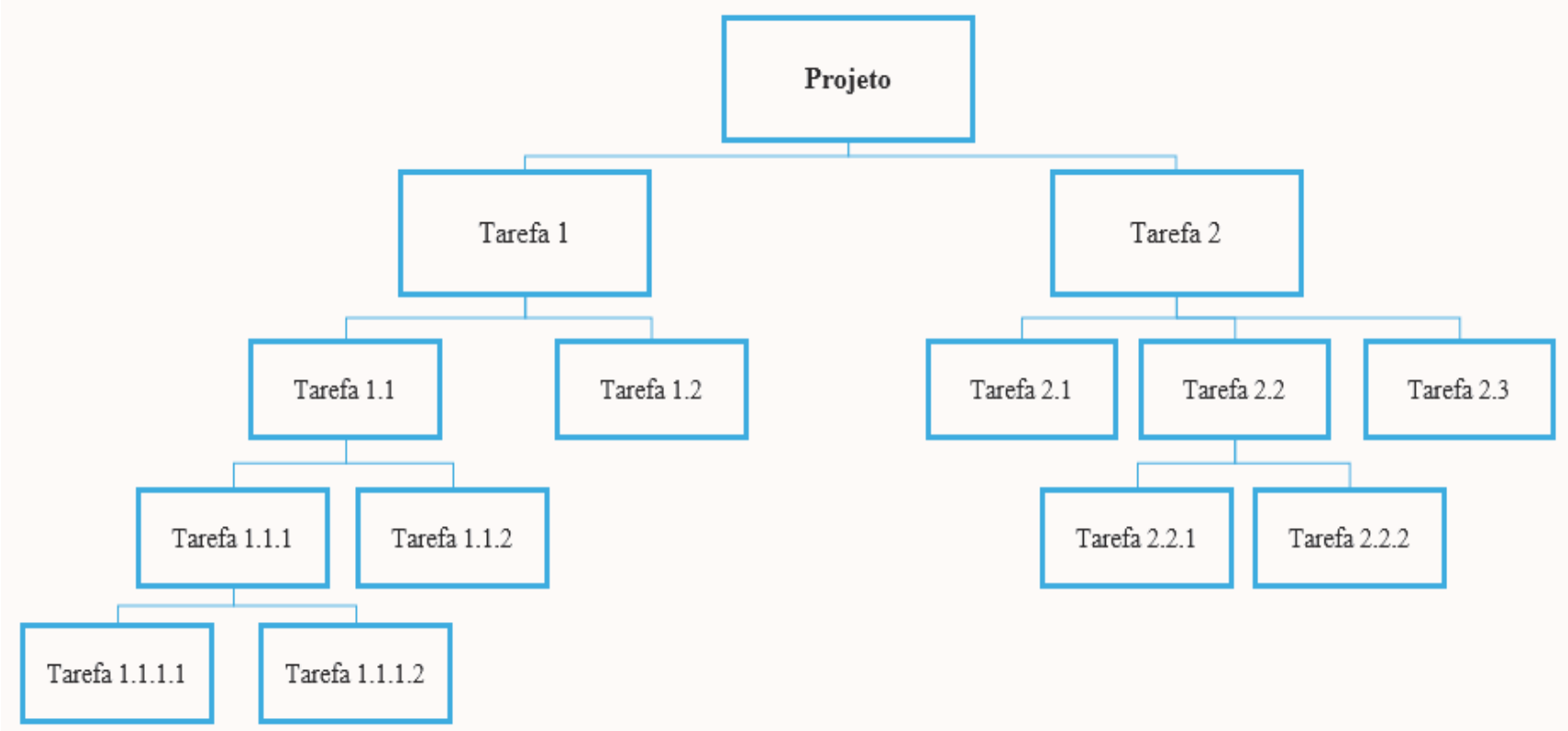

Figura 03 - Exemplo de EAP de projeto

Fonte: Autores

\section{PROCEDIMENTOS METODOLÓGICOS}

A metodologia utilizada nesta pesquisa consistiu na identificação dos subprojetos de um projeto de construção de usinas geradoras de energia elétrica de fonte renovável de forma geral, através de pesquisa bibliográfica como etapa inicial, seguida pela etapa de definição da ligação e relação entre os subprojetos e suas atividades com foco no impacto em atraso no cronograma final, tendo em vista a relação de sucessores e predecessores entre eles.

As premissas utilizadas para conversão dos dados do projeto em um grafo foram dadas pelos seguintes pontos: (1) vértices do grafo: são os subprojetos do projeto de construção de usina geradora de energia elétrica de fonte renovável; (2) arestas do grafo: são as relações de impacto de atraso entre os subprojetos dado a relação de sucessão entre eles, ou seja, no caso de haver relação de impacto em cronograma final, dado um atraso em um dos subprojetos analisados par a par.
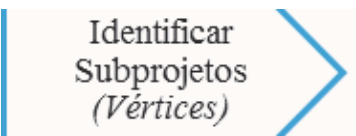
Relacionar
subprojetos
(Arestas)

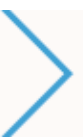
Desenhar
Grafo

Figura 04 - Fluxograma da metodologia Fonte: Autores
As etapas acima consistiram em representar o grafo formado pelos vértices e arestas em estudo, inserir os dados do grafo no software UCINET, calcular a centralidade de autovetor dos vértices e, por fim, analisar os resultados encontrados (BORGATTI; EVERETT; FREEMAN, 2002).

\section{RELAÇÃO DOS SUBPROJETOS DE CONS- TRUÇÃO DE USINA RENOVÁVEL EXPRESSO EM GRAFO}

Sabe-se que cada projeto é um evento específico e único com data de início e fim determinados, o que torna cada projeto com características próprias e distintas de outros projetos. Porém, de maneira geral nota-se a existência de características comuns e padrão dado uma determinada segmentação por tipo de projeto. No caso em estudo, um projeto de construção de usina geradora de energia elétrica de fonte renovável, como eólico, solar, hídrico e biomassa, possuem de forma geral a seguinte Estrutura Analítica de Projeto expressa na figura 05.

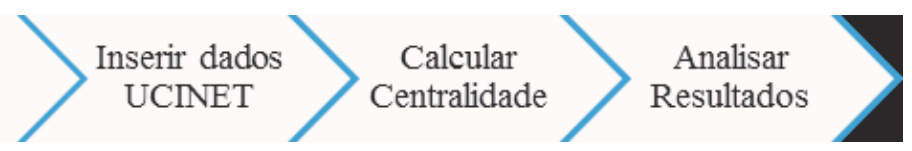




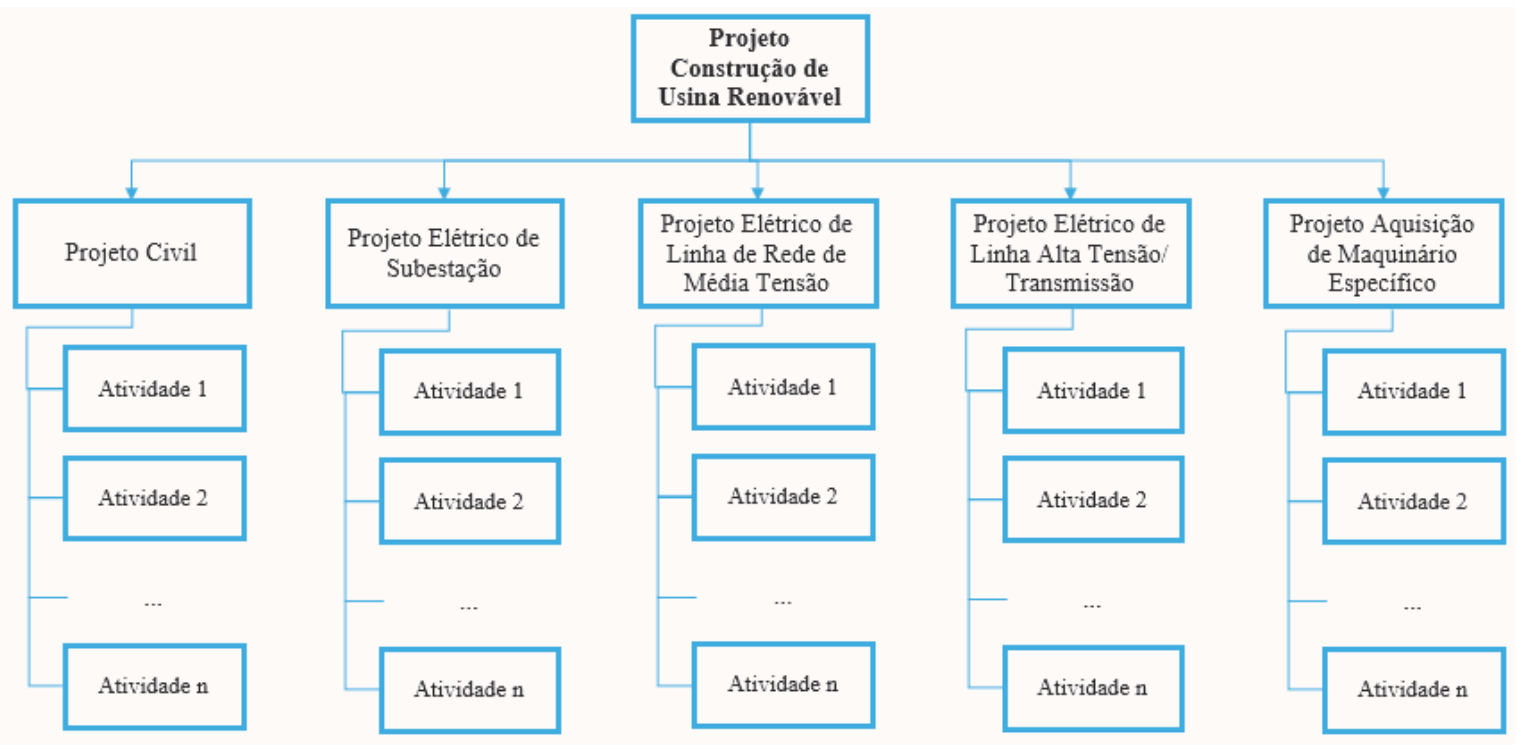

Figura 05 - EAP de projeto de construção de usina geradora de energia elétrica Fonte: Autores

\begin{tabular}{|l|l|}
\hline Subprojeto & Descrição \\
\hline (1) Projeto Civil & $\begin{array}{l}\text { Terraplanagem, construção e manutenção de acessos internos e externos, construção e manutenção } \\
\text { do canteiro de obras. }\end{array}$ \\
\hline (2) Projeto Elétrico de Subestação & $\begin{array}{l}\text { Construção da subestação e bay, construção e teste da Sala de Controle e Comunicação, incluindo o } \\
\text { fornecimento de geradores de material necessário. }\end{array}$ \\
\hline $\begin{array}{l}\text { (3) Projeto Elétrico de Linha de Rede de } \\
\text { Média Tensão }\end{array}$ & $\begin{array}{l}\text { Construção da linha de média tensão no interior do parque (lançamento de postes e cabos) desde o } \\
\text { maquinário gerador até a subestação própria. Incluindo a conexão e teste de energização. }\end{array}$ \\
\hline $\begin{array}{l}\text { (4) Projeto Elétrico de Linha de Alta } \\
\text { Tensão/Transmissão }\end{array}$ & $\begin{array}{l}\text { Construção da linha de alta tensão no exterior do parque (lançamento de torres e cabos) desde a subestação } \\
\text { própria até o bay de conexão da subestação da Distribuidora. Incluindo a conexão e teste de energização. }\end{array}$ \\
\hline $\begin{array}{l}\text { (5) Projeto de Aquisição de } \\
\text { Maquinário Específico }\end{array}$ & $\begin{array}{l}\text { Fornecimento, instalação e comissionamento dos maquinários geradores (Aerogeradores, turbina } \\
\text { elétrica hídrica, painéis solares/conversores). }\end{array}$ \\
\hline
\end{tabular}

Tabela 01 - Descrição dos subprojetos

Fonte: Autores

Como descrito anteriormente, os subprojetos abordados no estudo são a consolidação de atividades do projeto dado sua semelhança em escopo e objetivo final. A descrição dos subprojetos está detalhada na tabela 01 acima.

Ao transformarmos a relação dos cinco subprojetos em estudo em vértices, tomamos posse de nomenclatura por letras em ordem alfabética a fim de facilitar a representação gráfica. A tabela 02 a seguir demonstra o de-para entre os subprojetos e os vértices estabelecidos.

\begin{tabular}{|l|l|}
\hline Vértice & Descrição \\
\hline A & (1) Projeto Civil \\
\hline B & (2) Projeto Elétrico de Subestação \\
\hline C & (3) Projeto Elétrico de Linha de Rede de Média Tensão \\
\hline D & (4) Projeto Elétrico de Linha de Alta Tensão/ Transmissão \\
\hline E & Projeto de Aquisição de Maquinário Específico \\
\hline
\end{tabular}

Tabela 02 - Relação de vértices e subprojetos Fonte: Autores
A etapa seguinte consiste na representação gráfica dos vértices determinados no estudo e suas arestas que retratam a relação de impacto em atraso de prazo dos subprojetos dois a dois. Considerando de forma os impactos, num primeiro momento como uma relação direcionada, tem-se a seguinte representação gráfica ilustrada na figura 06 .

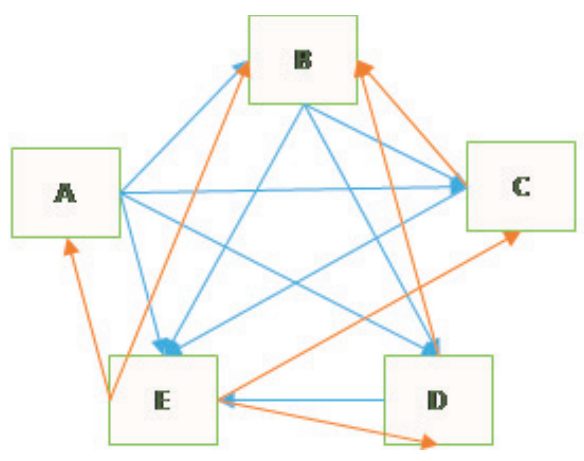

Figura 06 - Representação gráfica dos impactos em atraso dos subprojetos Fonte: Autores 
A descrição da relação de impacto em atraso em cronograma dos subprojetos dois a dois e uma breve justificativa desse impacto com base nas atividades que os compõem são detalhadas na tabela 03 após a seção 5 sobre resultados.

Dado que no presente estudo considera-se um grafo conexo simples e não direcionado (FAWCETT, 2006), tem-se o grafo final que representa o estudo conforme figura 07 a seguir. Sendo cada aresta a representação de impacto entre dois vértices independente do sentido e direção do mesmo.

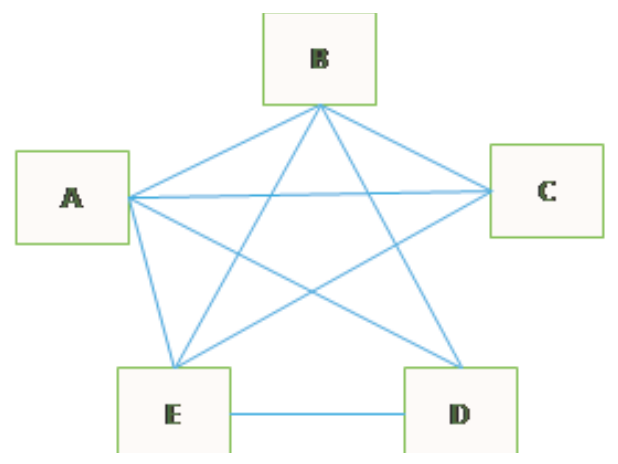

Figura 07 - Grafo em estudo Fonte: Autores

\section{RESULTADOS DO CÁLCULO DE CENTRALI- DADE DE AUTOVETOR}

Conforme descrito nos procedimentos metodológicos, utilizou-se do software UCINET para cálculo das centralidades de autovetor dos vértices em estudo. Através da ferramenta NetDraw, esse sistema também permite a representação gráfica dos vértices e arestas que forma o Grafo em estudo, conforme figura a seguir.

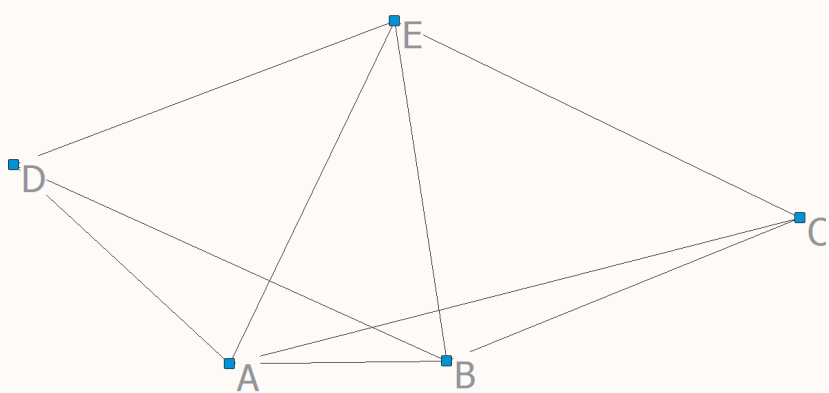

Figura 08 - Grafo plotado pelo NetDraw Fonte: Autores

O sistema UCINET funciona através da inserção dos dados do Grafo através da Matriz Adjacência do mesmo, ou seja, da matriz que representa a existência ou não de ligação entre os vértices dois a dois. Ao inserir tais dados, calcula-se as características do Grafo, entre elas a centralidade de autovetor conforme fórmula descrita no item 2.1.3.
Os autovetores calculados do grafo em estudo se dão conforme a figura 09 a seguir:

\section{Eigenvectors of MATRIZ ADJASCENCIA}

\section{1}

Eigen

vecto

$r$

1 A 0.479

2 B 0.479

3 C 0.394

4 D 0.394

5 E 0.479

5 rows, 1 columns, 1 levels.

Figura 09 - Autovetores do grafo em estudo Fonte: Autores

Pela centralidade de autovetor, dá-se que os vértices de maior índice são os nós mais centrais e impactantes no Grafo. No caso em estudo, representam os subprojetos cujos atrasos impactam mais o cronograma final do projeto total. São 3 os vértices de maior autovetor: (A) projeto civil; (B) Projeto Elétrico de Subestação e (E) Projeto de Aquisição de Maquinário Específico.

Tendo em vista o impacto do atraso do subprojeto no grafo representado pelo tamanho do vértice, tem-se a representação gráfica a seguir:

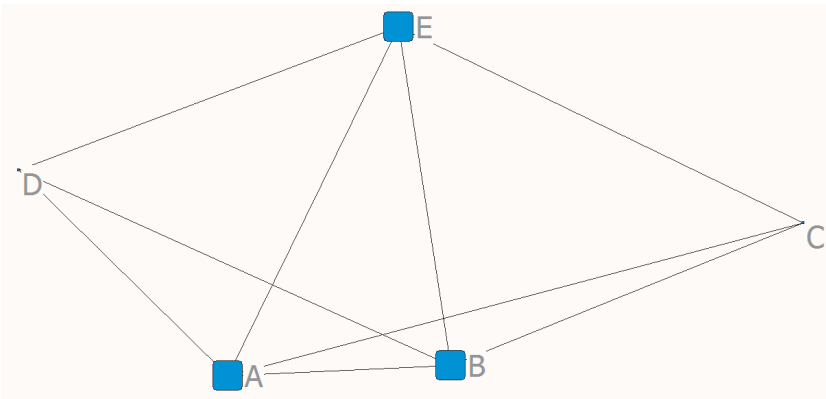

Figura 10 - Representação gráfica dos autovetores Fonte: Autores

O sistema UCINET traz como resultado da análise os dados característicos, como menor autovetor, maior autovetor, autovetor médio, desvio-padrão dos resultados encontrados, entre outros. Esses dados estão detalhados na figura a seguir extraída do relatório final do sistema. 


\section{Descriptive Statistics}

$\begin{array}{rrr} & & 1 \\ & \begin{array}{r}\text { Eigen } \\ \text { vecto }\end{array} \\ & r \\ & ---- \\ & & 0.394 \\ 1 & \text { Minimum } & 0.445 \\ 2 & \text { Average } & 0.479 \\ 3 & \text { Maximum } & 0.479 \\ 4 & \text { Sum } & 2.226 \\ 5 & \text { Standard Deviation } & 0.042 \\ 6 & \text { Variance } & 0.002 \\ 7 & \text { SSQ } & 1 \\ 8 & \text { MCSSQ } & 0.009 \\ 9 & \text { Euclidean Norm } & 1 \\ 10 & \text { Observations } & 5 \\ 11 & \text { Missing } & 0 \\ 12 & \text { Std. Deviation (n-1) } & 0.046 \\ 13 & \text { Variance (n-1) } & 0.002 \\ 14 & \text { Binary valued } & 0 \\ 15 & \text { Negatives } & 0 \\ 16 & \text { Integer valued } & 0 \\ 17 & \text { Weighted Obs } & 5 \\ 18 & & 0\end{array}$

18 rows, 1 columns, 1 levels.

Eigenvector centralization percentages

\section{1 \\ Plan1 \\ -...-. \\ 1 Centralization 12.004}

1 rows, 1 columns, 1 levels.

Figura 11 - Dados estatísticos do grafo em estudo

Fonte: Autores

Positive eigenvalues of MATRIZ ADJASCENCIA

$\begin{array}{rrrr} & 1 & 2 & 3 \\ & \begin{array}{r}\text { Eigenva } \\ \text { lue }\end{array} & \begin{array}{r}2 \\ \text { iance }\end{array} & \\ & & & \\ 1 & 3.646 & 100 & 100 \\ 2 & 0.000 & 0.000 & 100\end{array}$

2 rows, 3 columns, 1 levels.

Figura 12 - Dados estatísticos do grafo em estudo Fonte: Autores

\begin{tabular}{|c|c|}
\hline Aresta & Descrição da relação de atrasos \\
\hline$A B$ & $\begin{array}{l}\text { Atraso na terraplanagem e construção de acessos inter- } \\
\text { nos e externos (A) acarreta em atraso no início da obra de } \\
\text { construção da subestação (B) dado a impossibilidade de } \\
\text { acesso de transporte e pessoal por não conclusão civil. }\end{array}$ \\
\hline$A C$ & $\begin{array}{l}\text { Atraso na terraplanagem e construção de acessos internos } \\
\text { e externos (A) acarreta em atraso no início da obra de cons- } \\
\text { trução da rede de média tensão (C) dado a impossibilidade } \\
\text { de acesso de transporte e pessoal por não conclusão civil. }\end{array}$ \\
\hline$A D$ & $\begin{array}{l}\text { Atraso na terraplanagem e construção de acessos internos } \\
\text { e externos (A) acarreta em atraso no início da obra de cons- } \\
\text { trução da rede de alta tensão (D) dado a impossibilidade de } \\
\text { acesso de transporte e pessoal por não conclusão civil. }\end{array}$ \\
\hline $\mathrm{AE}$ & $\begin{array}{l}\text { Atraso na terraplanagem e construção de acessos internos } \\
\text { e externos, além de atrasos na concretagem de bases (A) } \\
\text { acarreta em atraso no início da instalação do maquinário } \\
\text { (E) dado a impossibilidade de acesso de transporte e pes- } \\
\text { soal por não conclusão civil. } \\
\text { Atraso no comissionamento e testes dos maqui- } \\
\text { nários (E) acarreta em atraso na manutenção de } \\
\text { acessos internos, externos e canteiro (A). }\end{array}$ \\
\hline BC & $\begin{array}{l}\text { Atraso na finalização da subestação (B) acarreta em atraso } \\
\text { da conexão e testes da linha de média tensão (C). } \\
\text { Atraso na finalização da linha de média tensão (C) acar- } \\
\text { reta em atraso na conexação e testes da subestação (B). }\end{array}$ \\
\hline $\mathrm{BD}$ & $\begin{array}{l}\text { Atraso na finalização da subestação (B) acarreta em atraso } \\
\text { da conexão e testes da linha de alta tensão (D). } \\
\text { Atraso na finalização da linha de alta tensão (D) acarreta } \\
\text { em atraso na conexação e testes da subestação (B). }\end{array}$ \\
\hline $\mathrm{BE}$ & $\begin{array}{l}\text { Atraso na finalização da subestação (B) acarreta em atraso } \\
\text { do comissionamento dos maquinários geradores (E). } \\
\text { Atraso no comissionamento e testes dos maquinários (E) } \\
\text { acarreta em atraso na conexação e testes de subestação (B). }\end{array}$ \\
\hline CE & $\begin{array}{l}\text { Atraso na finalização da linha de média tensão (C) acarreta em } \\
\text { atraso do comissionamento dos maquinários geradores }(E) \text {. } \\
\text { Atraso no comissionamento e testes dos maquinários (E) } \\
\text { acarreta em atraso na conexação e testes na rede de mé- } \\
\text { dia tensão (C). }\end{array}$ \\
\hline $\mathrm{DE}$ & $\begin{array}{l}\text { Atraso na finalização da linha de alta tensão (D) acarreta em } \\
\text { atraso do comissionamento dos maquinários geradores (E). } \\
\text { Atraso no comissionamento e testes dos maquinários (E) } \\
\text { acarreta em atraso na conexação e testes da rede de alta } \\
\text { tensão (D). }\end{array}$ \\
\hline
\end{tabular}

Tabela 03 - Descrição dos impactos de atraso em cronograma entre subprojetos Fonte: Autores

\section{CONCLUSÃO}

O estudo da aplicação de Teoria dos Grafos através dos conceitos de centralidade de autovetor em projetos de construção de usinas de geração de energia elétrica de fontes renováveis evidenciou a aplicabilidade do método no setor estudado.

Os vértices foram tidos como os subprojetos principais do projeto de construção; e as arestas a relação entre os subprojetos dados impactos em atraso de cronograma físico por interdependência de atividades e relação de sucessão entre as mesmas.

O grafo formado através dessa análise para estudo e aplicação do método, possui cinco vértices e nove arestas, sendo três vértices de grau quatro e dois vértices de grau três. 
O cálculo da centralidade de autovetor apontou que os vértices que possuem os maiores graus são também os vértices de maior autovetor, e, portanto, os vértices de maior centralidade estudada. A centralidade de autovetor é a indicada para medir a rapidez na propagação de um efeito na rede de grafos.

Logo, os subprojetos representados pelos três vértices de maior centralidade são os responsáveis pela maior propagação de atraso no cronograma físico final do projeto. $E$, com isso, devem ser gerenciados e controlados atentamente a fim de mitigar atrasos nos mesmos para que o cronograma final acordado do projeto não apresente atraso significativos.

Por fim, como principais limitações percebidas tem-se a modelagem, que considera aspectos dos subprojetos como prováveis problemas na execução dos mesmos no canteiro de obras. No mais, como proposta para estudos futuros pode ser citado a combinação da com análise de grafos com análises tradicionais de cronograma, como por exemplo, o gráfico de Gantt para melhor visualização de "gaps" e oportunidades de melhoria.

\section{REFERÊNCIAS}

BEAUCHAMP, M. A. An improved index of centrality.
2010. 111 f. Dissertação (Mestrado em Engenharia de Produção) - Universidade Federal do Rio de Janeiro, Programa de Engenharia de Produção, Rio de Janeiro, 2010.

DEL-VECCHIO, R. R. et al. Medidas de centralidade da teoria dos grafos aplicada a fundos de ações no Brasil. In: XLI SIMPÓSIO BRASILEIRO DE PESQUISA OPERACIONAL. Anais... Porto Seguro, 2009.

FAWCETT, T. ROC graphs with instance-varying costs. Pattern Recognition Letters, v. 27, n. 8, p. 882-891, 2006.

FREEMAN, L. C., 1978/79, “Centrality in Social Networks: Conceptual Clarification", Social Networks, v. 1, pp. 215-239.

GRASSI, R.; STEFANI, S.; TORRIERO, A. Centrality in organizational networks. International Journal of Intelligent Systems, v. 25, n. 3, p. 253-265, 2010. NETTO, P. O. B. Grafos: teoria, modelos, algoritmos. São Paulo: Edgard Blücher, 2003.

PMBOK, G. Um guia do conjunto de conhecimentos em gerenciamento de projetos. Project Management Institute. Anais... Newtown, 2004.

SLACK, N. et al. Administração da Produção. 3 ed. São Paulo: Atlas, 2009.
2, p. 161-163, 1965.

BONACICH, P. Power and centrality: A family of measures. American journal of sociology, v. 92, n. 5, p. 1170-1182, 1987.

BONDY, J. A.; MURTY, U. S. R. Graph theory with applications. [s.I.] Macmillan London, 1976. v. 290 BORBA, E. M. Medidas de centralidade em grafos e aplicações em redes de dados. 2013. $77 \mathrm{f}$. Dissertação (Mestrado em Matemática Aplicada) Universidade Federal do Rio Grande do Sul, Programa de Pós-Graduação em Matemática Aplicada, Porto Alegre, 2013.

BORGATTI, S. P. Centrality and network flow. Social networks, v. 27, n. 1, p. 55-71, 2005.

BORGATTI, S. P.; EVERETT, M. G.; FREEMAN, L. C. Ucinet for Windows: Software for social network analysis. Harvard, MA: Analytic Technologies, 2002.

BRACIANI, U. Estrutura de custos para implantação das usinas de geração de energia elétrica no Brasil. 2011. 84 f. Monografia (Bacharel em Ciências Econômicas) - Departamento de Ciências Econômicas, Universidade Federal de Santa Catarina, Florianópolis, 2011.

DE FREITAS, L. Q. Medidas de centralidade em grafos. 


\section{AUTORES}

LETÍCIA FERNANDES BELLA GOMES, M.Sc. | Universidade Federal Fluminense | Engenharia de Produção | Niterói, RIO DE JANEIRO (RJ) - Brasil | Correspondência para: R. Passo da Pátria, 152-470 - São Domingos, Niterói - RJ, 24210-240 | E-mail: leticiafbella@gmail.com

KARINE FÁTIMA DE FREITAS | Universidade Federal Fluminense | Engenharia de Produção| Rio das Ostras, RIO DE JANEIRO (RJ) - Brasil | Correspondência para: R. Recife, Lotes 1-7 Jardim Bela Vista, Rio das Ostras -RJ, 28895532 | E-mail: karinefreitas@id.uff.br

ORCID: https://orcid.org/0000-0003-2212-1789

RICARDO LUIZ FERNANDES BELLA, Dr. | Universidade Federal Fluminense | Engenharia de Produção | Rio das Ostras, RIO DE JANEIRO (RJ) - Brasil | Correspondência para: R. Recife, Lotes 1-7 - Jardim Bela Vista, Rio das Ostras -RJ, 28895532 | E-mail: ricardobella@id.uff.br

ORCID: https://orcid.org/0000-0003-1400-2309

RENATA RAPOSO DEL-VECCHIO, Dra. | Universidade Federal Fluminense | Matemática | Niterói, RIO DE JANEIRO (RJ) Brasil | Correspondência para: R. Passo da Pátria, 152-470 - São Domingos, Niterói - RJ, 24210-240 | E-mail: rrdelvecchio@id.uff.br

ORCID: https://orcid.org/0000-0001-6741-2403

GILSON BRITO ALVES LIMA, Dr. | Universidade Federal Federal | Engenharia de Produção | Niterói, RIO DE JANEIRO (RJ) Brasil | Correspondência para: R. Passo da Pátria, 152-470 - São Domingos, Niterói - RJ, 24210-240 | E-mail: glima@ id.uff.br

\section{COMO CITAR ESTE ARTIGO}

GOMES, Letícia Fernandes Bella; FREITAS, Karine Fátima de; BELLA, Ricardo Luiz Fernandes; DEL-VECCHIO, Renata Raposo; LIMA, Gilson Brito Alves.Centralidade de Grafos Aplicada À Projetos de Energia Renovável. MIX Sustentável, [S.I.], v. 6, n. 4, p. 105-114, ago. 2020. ISSN 24473073. Disponível em:<http://www. nexos.ufsc.br/index.php/mixsustentavel>. Acesso em: dia mês. ano. doi:https://doi.org/10.29183/2447-3073. MIX2020.v6.n4.105-114. 
